\title{
APPLICATION OF LATTICE-GAS MODEL TO THE SIMULATION OF COMPLEX ECONOMICAL SYSTEMS
}

\author{
Tjipto Juwono \\ Department of Green Economy, Surya University \\ tjipto.juwono@surya.ac.id
}

\begin{abstract}
The lattice-gas model simulations are used to reproduce the collective behavior of complex economic systems. By correlating different manifestation of the collective behaviors to the parameter changes in the simulation, we identify the underlying mechanism of the various manifestation of the collective behavior. The standard growth model is reproduced by the model with non-interacting agent. As we introduce interacting agents, the growth model departs from the standard. Using cluster labeling algorithm we obtain number density histograms and diversity to explain the underlying mechanism of the growth dynamics.
\end{abstract}

Keywords: lattice-gas model, complex economic systems, mechanism, cluster labeling algorithm

Simulasi lattice-gas model digunakan untuk mereproduksi perilaku kolektif dari sistem ekonomi kompleks. Dengan mencari korelasi antara manifestasi perilaku kolektif dengan variasi parameter pada simulasi, kita mengidentifikasi mekanisme di balik perilaku kolektif tersebut. Model pertumbuhan standard dihasilkan oleh model dengan agen-agen yang tidak saling berinteraksi. Introduksi agen-agen yang saling berinteraksi menyebabkan model pertumbuhannya bergeser dari model standard. Algoritma cluster-labeling lalu digunakan untuk memperoleh histogram kerapatan jumlah cluster dan ukuran-keanekaragaman yang digunakan untuk menjelaskan mekanisme di balik dinamika pertumbuhan yang terjadi.

Kata kunci: lattice-gas model, sistem ekonomi kompleks, mekanisme, algoritma cluster-labeling

\section{Introduction}

People use equations (often called "models") to explain collective behaviors in a complex system without taking into account the underlying mechanisms that generate the collective behavior. While quite useful and powerful, the equations may fail to explain the microscopical mechanisms behind them.

The collective behavior, however, change from time to time. The equations that work for some collective behaviors may not work for some other collective behaviors. If a collective behavior persists for a significant period of time, the equations that describe the collective behavior will be very useful to predict the future behavior of the system. However, the equations themselves do not predict the change in collective behavior that have to be explained by different equations. The situation can get worse when the collective behavior has a stochastic element in it.

In this study we propose a method to connect the microscopic dynamics of a system with its collective behavior. While the reality is much more complex than the model system that we use, our model is useful to highlight the dominant microscopical features that would lead to the collective 
behavior. The purpose of the simulation is, therefore, to explain the collective behavior in term of its underlying microscopic behavior. We employ a simple lattice-gas model where it is simple enough to measure the collective behavior on one hand and to describe the microscopical behavior on other hands, and to make a direct connection between the two.

Lattice-gas model has been used extensively in statistical physics, surface physics, and electrochemistry. Here we propose to apply it to complex economical systems. In this study we examine the dynamics of distributions of particles adsorbed on a surface. The dynamics of particle distributions can be viewed as the change of configurations with time. The configurations are created by adsorption, desorption, or diffusion.

This kind of study is not only important for surface-science studies, but also very useful in the study of complex system in general. The average properties of the lattice-gas surface as a function of time are used to model the collective behavior of complex economical systems. We measure the average properties such as coverage and correlation length to represent the collective behavior. The microscopic configurations are representated by snapshots that includes the histogram of size distributions and configuration snapshots, coupled with diversity measurements.

Lattice-gas model is the simplest kind of model for adsorption, desorption, and diffusion on a surface. The term "lattice gas" was first introduced by Yang and Lee (Yang and Lee, 1952) to describe a collection of atoms, which can occupy only discrete positions in space that constitute a lattice structure. We limit our study to systems in which each site can be occupied by at most one atom. In this model, we define the occupancy number $c_{i j}$ as in Equation 1 below:

$$
c_{i j}= \begin{cases}1 & \text { when site }(i, j) \text { is occupied } \\ 0 & \text { otherwise }\end{cases}
$$

Equation 1

The surface is then modeled by a lattice where each site is marked as either occupied or unoccupied. Therefore, adsorption is the process of occupying an empty lattice site by a particle, while desorption is the opposite. Diffusion can be viewed as desorption from an occupied site followed by adsorption on a neighboring site. In our study, we work with square lattices with a total number of sites $N=L \times L$ with periodic boundary conditions.

The total number of adsorbed particles is given by Equation 2:

$$
N_{p}=\sum_{i=1}^{L} \sum_{j=1}^{L} c_{i j} .
$$

Equation 2 
The coverage (or particle concentration) is defined as in Equation 3:

$$
\theta=\frac{N_{p}}{N}
$$

Equation 3

In our modeling of an economic system, a particle is a representative of an agent. When a particle occupies a site, it is basically representing a contribution to economic growth. When a particle leave a site, it is representing a contribution to economic shrinkage. The total growth is proportional to the change of coverage, and the rate would depend on whether the particles are interacting with each other or not. And if they are interacting with each other, how exactly the interactions manifest themselves. The interaction (or non-interaction) creates the dynamics of surface morphology, which later can be shown by diversity and size distribution dynamics. By measuring this morphological dynamics and correlates it with the average properties of the system as a function of time, we effectively connect the microscopical dynamics to the collective behavior.

\section{Growth Model for Non-interacting Agents}

In the simplest approach, the particles are not mutually interacting. The only limitation is that each lattice site can be occupied by at most one particle at a time. In this case, the way to adsorb, desorp, or diffuse particles on the surface is through random adsorption, desorption, or diffusion. Any application of additional rules for the particle distribution requires some sort of interaction.

The objective is to create a series of random configurations, starting from an empty lattice up to a coverage cut-off close to $\theta=1.0$. To achieve this, we use the simplest way of creating random configurations, which is random adsorption. The algorithm for random adsorption is as follows:

1. Start from an empty lattice,

2. Choose a lattice site randomly,

3. If the site is unoccupied, place a particle. If not, do nothing,

4. For each attempt, increase time with one unit regardless whether the attempt is successful or not.

We set up a square lattice, with $L=256$. We run 100 simulations in which we record the time interval for every fixed interval of $\theta$ and average the results ${ }^{1}$. Figure 1 shows a plot of coverage versus time for random distribution after averaging over 100 runs. Here the unit of time is one Monte Carlo Steps per Site (MCSS), where one MCSS means that each site is visited one time on average. For a lattice of size $N=L^{2}$, one MCSS is equal to $L^{2}$ attempts. The figure shows that the time to increase the coverage by a given amount increases with increasing coverage, as the attempt to find an empty site is becoming more difficult. The function $\theta(t)=1-\exp (-t)$ fits well to the simulation result. This is 
verified theoretically as follows. We assume that the change of the number of occupied sites $d N / d t$ is proportional to available empty sites at time $t$,

$$
\frac{d N}{d t}=k\left(L^{2}-N\right)
$$

Equation 4

multiply both sides with $1 / L^{2}$,

$$
\frac{d \theta}{d t}=k(1-\theta)
$$

\section{Equation 5}

and integrate to obtain:

$$
\theta(t)=1-\exp (-k t)
$$

Equation 6
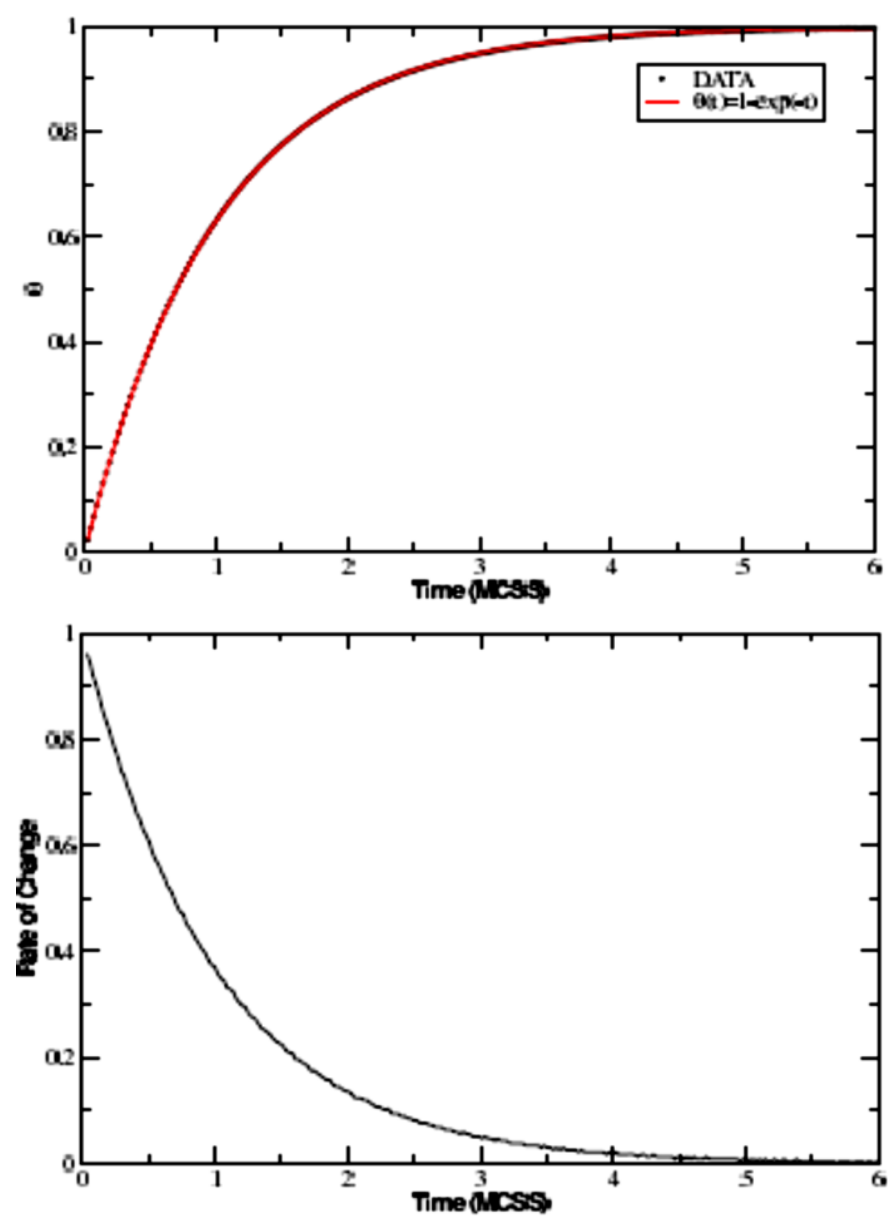

Figure 1. Coverage, $\theta(t)$ for random adsorption. The adsorption is faster at the beginning and becomes slower as the number of empty sites decreases, hence making the attempt to adsorb more difficult. The lower panel shows that the rate of change is decreasing with time. This is in accordance with some known growth model, such as Solow Growth model. The theoretical function $\theta(t)=1-\exp (-t)$ fits well to the result of the simulation. 
As shown in Figure 1, fitting the function to the data gives $k=1$ as expected. Figure 2 gives a few snapshots configurations at several coverages.

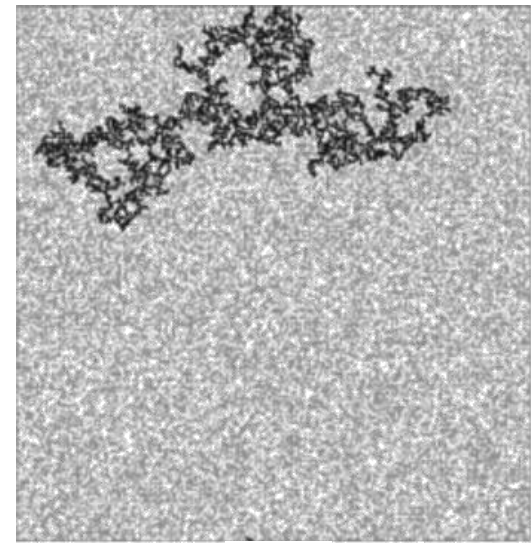

A

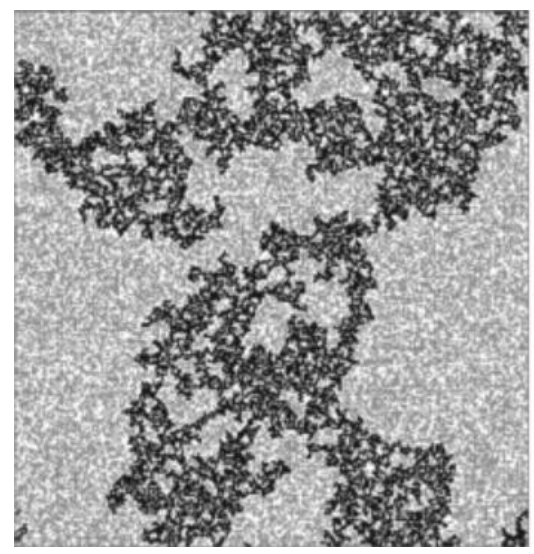

$\mathrm{C}$

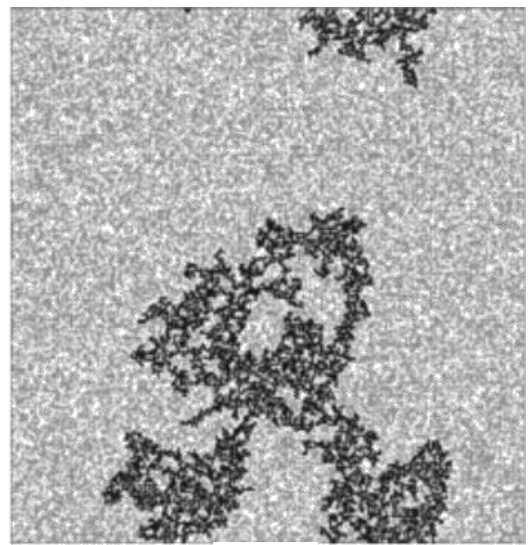

$\mathrm{B}$

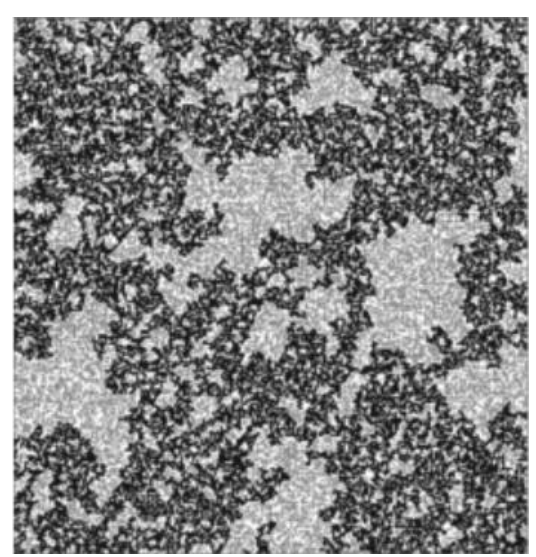

$\mathrm{D}$

Figure 2. Some snapshots of random distributions, taken at: (A) $\theta=0.58$, (B) $\theta=0.59$, (C) $\theta=0.60$, (D) $\theta=0.61$

As a visual aid, we colored the largest cluster dark, which gives us a rough idea about the sizes of the clusters. The size of the largest cluster increases slowly until the system reaches a coverage window where a spanning cluster suddenly appears. Thereafter, the whole lattice is dominated by the largest cluster.

\section{Growth Model for Interacting Agents}

In a complex economical system, the interaction between the agents plays a very important role. The difference between classical and modern economical model is basically the difference between incorporating or not-incorporating this interaction within the model. In our lattice-gas model, particles are being adsorbed or desorped with a certain profitability. The interactions, therefore, are manifested through these probabilities. 
Here we model attractive nearest-neighbor interactions using a simple rule. We calculate the energy $\triangle E$ of interaction with the nearest neighbors, and adsorb or desorp the particle with probability

$$
P=\frac{e^{-\Delta E / T}}{1+e^{-\Delta E / T}}
$$

\section{Equation 7}

Here, $T$ is the temperature of the system. In our economic modeling, the temperature measure the degree of the lateral mobility of the economic agents. When the temperature is high, the agents have higher probability to detach from a cluster of agents and attach to another cluster. Since the agents keep on detaching and attaching themselves, then it is more difficult for the system to form larger clusters. At higher temperatures, the mean size of the clusters are smaller. Obviously, at lower temperatures the mean size of the clusters is larger.

We do simulations for lattice size of $L=256$, at temperatures of $T=1.5,2.5,3.510 .0$. Figure 3 shows configuration snapshots for $\theta=0.35$.

Figure 4 shows the Growth Model for interacting agents. Interaction between agents clearly shift the usual model where the rate of change is simply decreasing. This classical model is represented by the results for very high temperature $(T=10.0)$. As we lower the temperatures, the effect of interaction becomes more apparent, and we see a departure from classical growth model. The rate of changes increase at the beginning until they reach the maximum, after which they start to decrease.

To understand more about the relation between the morphology and dynamics, we need information about the number density histograms. To have those, we need to do cluster labeling.

\section{Cluster Labeling}

One important measurement on a configuration is cluster counting in which we count the number of clusters and list the size of each cluster. From this list, we extract mean cluster size, cluster size distribution, diversity, and many other quantities. To generate this list, we employ the HoshenKopelman algorithm of cluster labeling (Hoshen and Kopelman, 1976). To execute the algorithm we need three main arrays: (1) site-label array $1(i, j)$ (Eq. 8), (2) cluster label array u( $i, j)$ (Eq. 9), and (3) proper label array $\mathrm{p}(l)($ Eq. 10).

The algorithm is as follows:

$$
\begin{aligned}
& \mathrm{l}(i, j)=\left\{\begin{array}{l}
-1 \text { when site }(i, j) \text { is occupied, } \\
0 \text { otherwise }
\end{array}\right. \\
& \text { Equation } 8 \\
& \mathrm{u}(i, j)=\left\{\begin{array}{l}
\text { cluster label, when site }(i, j) \text { is occupied, } \\
0 \text { otherwise. }
\end{array}\right.
\end{aligned}
$$

Equation 9 


$$
\mathrm{p}(l)=\left\{\begin{array}{c}
\text { next lower cluster label when the label } \\
l \text { belong to a multilabeled cluster, } \\
0 \text { when the label } l \text { is the lowest label of the cluster. }
\end{array}\right.
$$

Equation 10

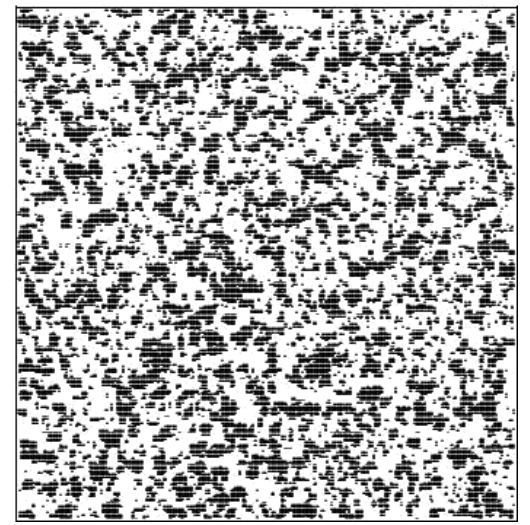

A

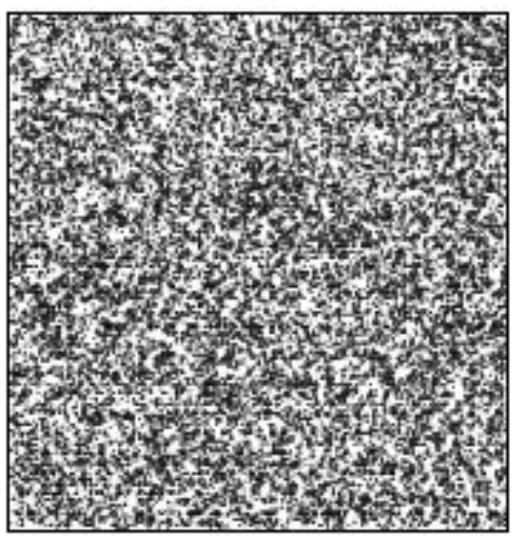

$\mathrm{C}$

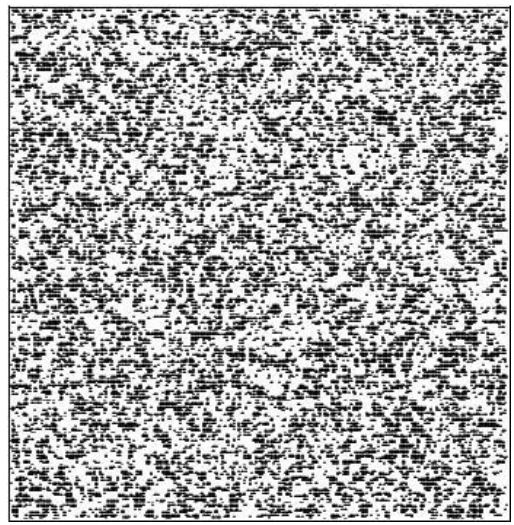

$\mathrm{B}$

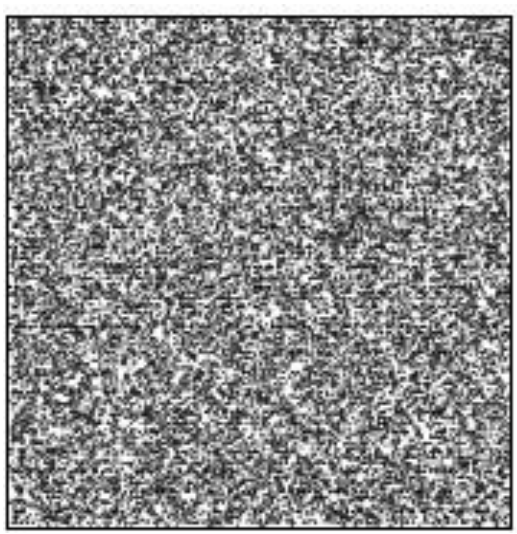

$\mathrm{D}$

Figure 3. Some snapshots for adsorptions, taken at $\theta=0.35$ for (A) $T=1.5$, (B) $T=2.5$, (C) $T=3.5$, (D) $T=10.0$. The lower temperature shows larger mean cluster size, higher temperature shows smaller cluster size.

1. Start by labeling the bottom left corner of the lattice by zero if it is unoccupied and 1 if it is occupied with the help of the site-label array $1(i, j)$. Scan the first row to the right. If an occupied site is connected to the previous occupied site, assign the same label as the previous site. If not, assign a new label. Store the label in the cluster label array $u$.

2. Scan the first column of the lattice the same way, starting from the second row going up to the last. Store the label in the cluster label array u.

3. Scan the remainder of the lattice row by row starting from the second row. Only this time we compare each site with the site to the left and below. Use $m=l(i-1, j) \times l(i, j-1)$ to check if both 
sites are occupied. If $m=0$, we continue the scan, and if $m=1$ we proceed to check the labels of the two sites. If they are different, choose the lowest label, and store it in the cluster label array $u$.

4. Scan the lattice for the second time, this time identifying clusters that have more than one label, and relabel the cluster by the lowest label with the help of the proper label array $p$. Also identify and correct the label of clusters separated by the edges, thus taking care of the periodic boundary conditions. Store the corrected labels in the cluster label array $u$.

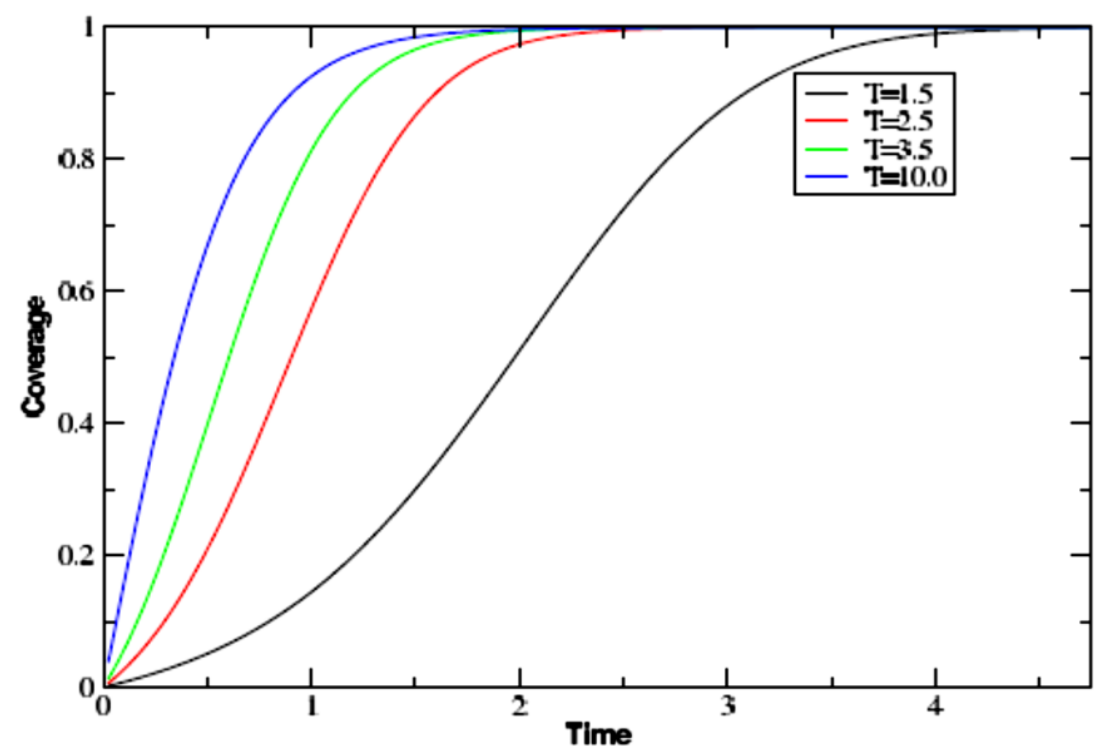

(A)

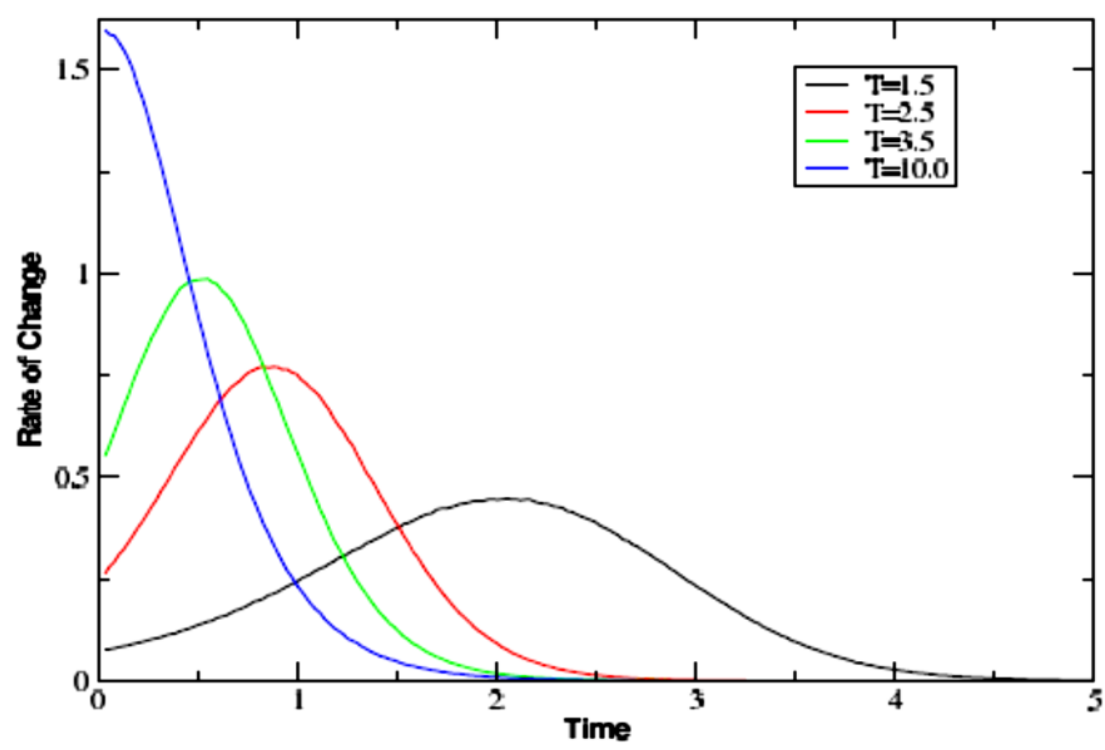

(B)

Figure 4. (A) Coverage $\theta(t)$ for Growth Model with $T=1.5,2.5,3.5,10.0$. (B)The rates are changing shapes as we lower the temperatures. 
Figure 5 shows a cluster that has more than one label (in this case, the lowest label is 2). The dark colored part of the cluster shows the part where multiple labels start to appear. Because we are labeling this cluster row by row (in this case we start from the bottom) and check the site to the left and below, the disconnected site to the right will be assigned a new label. Because of this new label, there is a chance that we will encounter a site where the left and below sites have different labels. Here, we choose the lowest of the two, and proceed with the labeling procedure until the whole cluster is labeled. The end result is a cluster that has more than one label.

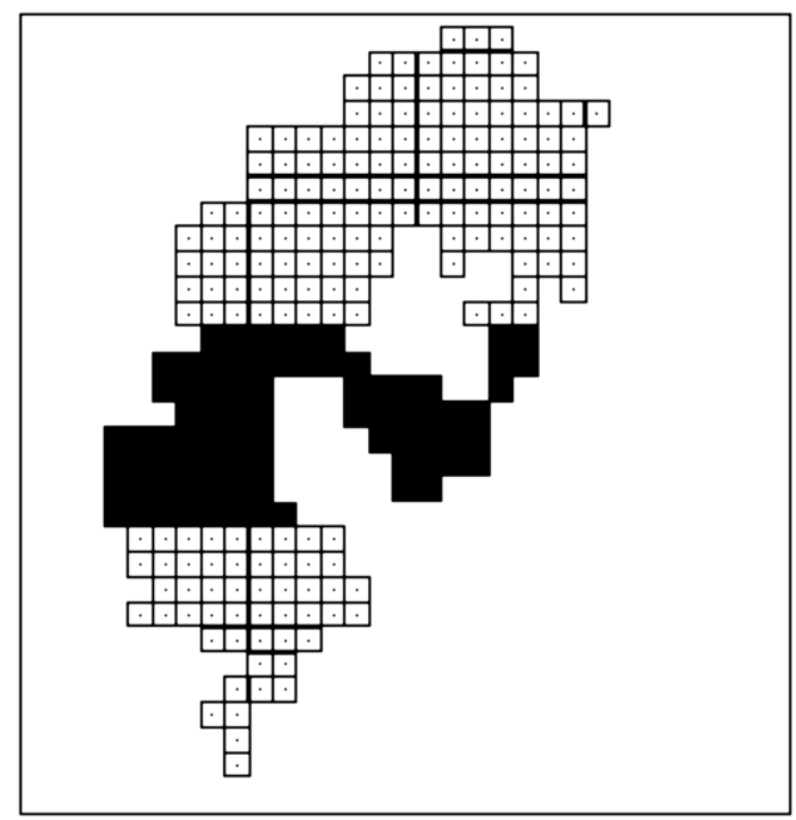

(A)

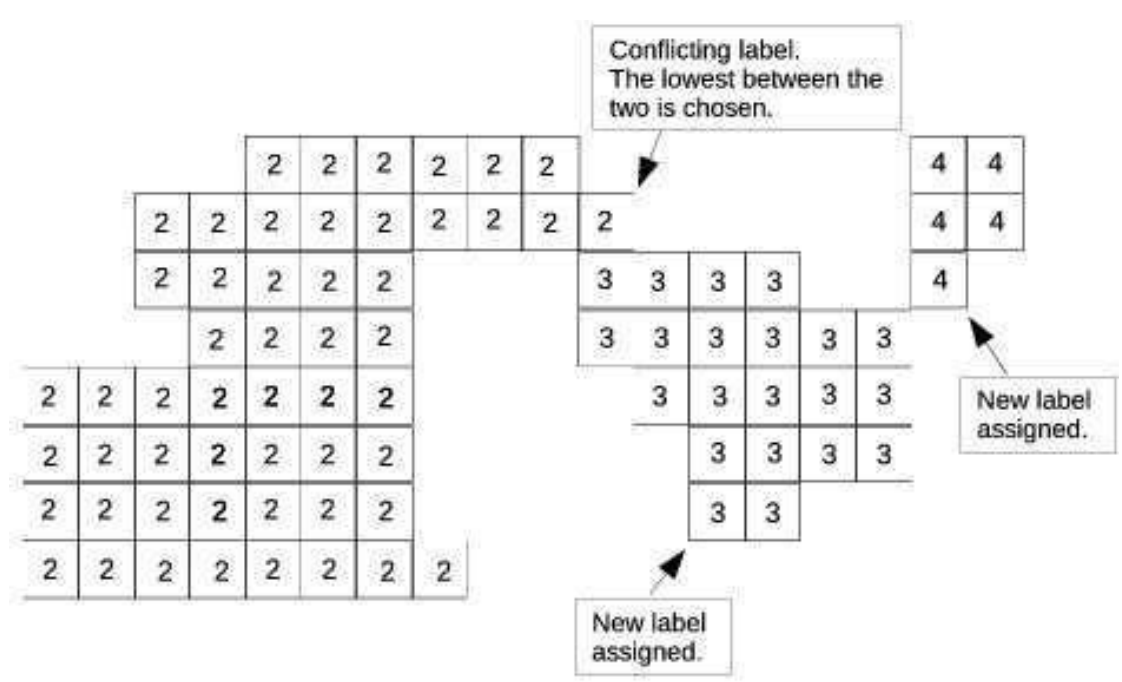

(B)

Figure 5. (A) a single cluster that has more than one label. (B) a portion of the cluster in (A), where we show examples of how the multilabeling starts. 
To correct the multilabel, we create an array of proper labels (Eq. 10). The initial value of the elements of this array is zero. And if all clusters have been labeled properly, the value of all the elements should be zero or stay zero. At the second scan, when we encounter a cluster with multilabels, the element of the proper label array corresponding to the site with the lowest label is set to zero. The element for the site corresponding to the next label is assigned with the lowest label, and the next with the second lowest, and so forth. For example, if a cluster has three labels, 2, 3 and 4, the corresponding proper label array elements would be: $\mathrm{p}(4)=3, \mathrm{p}(3)=2$, and $\mathrm{p}(2)=0$.

By means of this proper label array, the lowest label for each multilabeled cluster can be found and the cluster can immediately be relabeled properly. During the relabeling process, the cluster size corresponding to each proper label is counted, and after the counting the corresponding labels in the cluster label array $u$ are labeled properly, and the corresponding elements in the proper label array $p$ are set to zero. Now the cluster label array (Eq. 9) contains the proper labels and we can generate the cluster size list, and since the indices of this array are the coordinates of each site, this array is also useful for many cluster calculations (e.g. finding the spanning cluster).

\section{Number Density Histogram Plot}

The details of the configurations at the beginning and during the desorption are elaborated further from number density $\left(\rho_{s}\right)$ histogram plots (Frank et al. [2005]). To get the distribution we measure first the number density $n_{s}$ where $n_{s}$ is the number of the clusters with size s per site.

We average each result over 100 runs, therefore $n_{s}$ becomes the probability of finding on a randomly chosen site the center of a cluster of a size $s$. We then create the histogram for the number density distribution. The number density for larger clusters is very low compared to that of the small clusters. For that reason we use exponentially growing bins for the histograms. The growing bins are set up such that the next bin is always twice as large as the previous one. This results in an equal distribution of data points on a logarithmic scale. Figure 6 is an example of $n_{s}$ from a simulation with $T$ $=1.5,2.5,3.5,10.0$. Here we can see that reducing the temperatures increases the size and the number of the larger cluster and decreases the number of the smaller clusters, including the number of monomers. Such a change affects significantly the character of the growth model. The morphology with larger clusters results in a growth that start with an increase of rate of change in the beginning, reach the maximum, then start to decrease. 


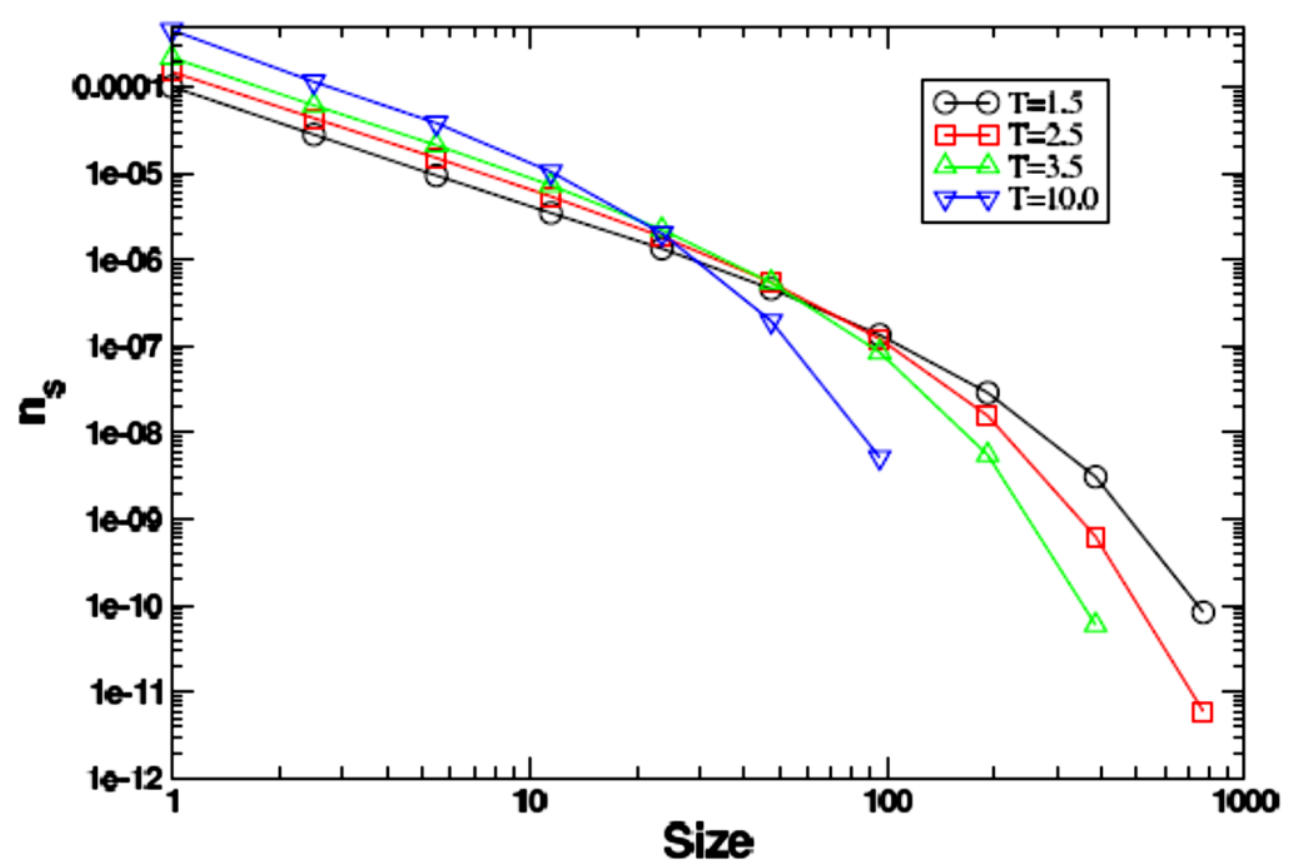

Figure 6. An example of $\rho_{s}$ from the simulation with $T=1.5,2.5,3.5,10.0$.

As the number of larger clusters is decreasing, the system reach the maximum of the rate of change faster. At very high temperature, the mean size of the cluster is small which results in a growth model where the rate of change is simply decreasing all the time.

\section{Diversity}

Other morphological measure that we can obtain by using the cluster labeling algorithm is diversity. We define $N(s, \theta)$ as the total number of clusters with size $s$ for a configuration with coverage $\theta$. The total number of clusters, averaged over the number of experiments, where each experiment results in a configuration with coverage $\theta$, is then (Tsang and Tsang, 1999):

$$
n(\theta)=\left\langle\sum_{s} N(s, \theta)\right\rangle .
$$

\section{Equation 11}

From Eq. 11, we define the diversity as (Tsang and Tsang, 1999):

$$
D(\theta)=\left\langle\sum_{s} \Theta[N(s, \theta)]\right\rangle .
$$

Equation 12

In the above equation, the Heaviside function is defined $\quad \mathrm{a} \Theta x)=1$ if $x>0$ and $\quad \Theta)=0$. Thus, $D(\theta)$ is the number of different cluster sizes for a given $\theta$. Figure 7 gives an example of $D(\theta)$ from a simulation with simple rule, with $T=1.5,2.5,3.5,10.0$. The peak of the diversity $D(\theta)$ for a random 
configuration gives us the maximum diversity that can be achieved for a particular square lattice size (in our case $L=256$ ). As we introduce correlations into the simulation, the maximum diversity is reduced, and the maximum is reached for lower $\theta$.

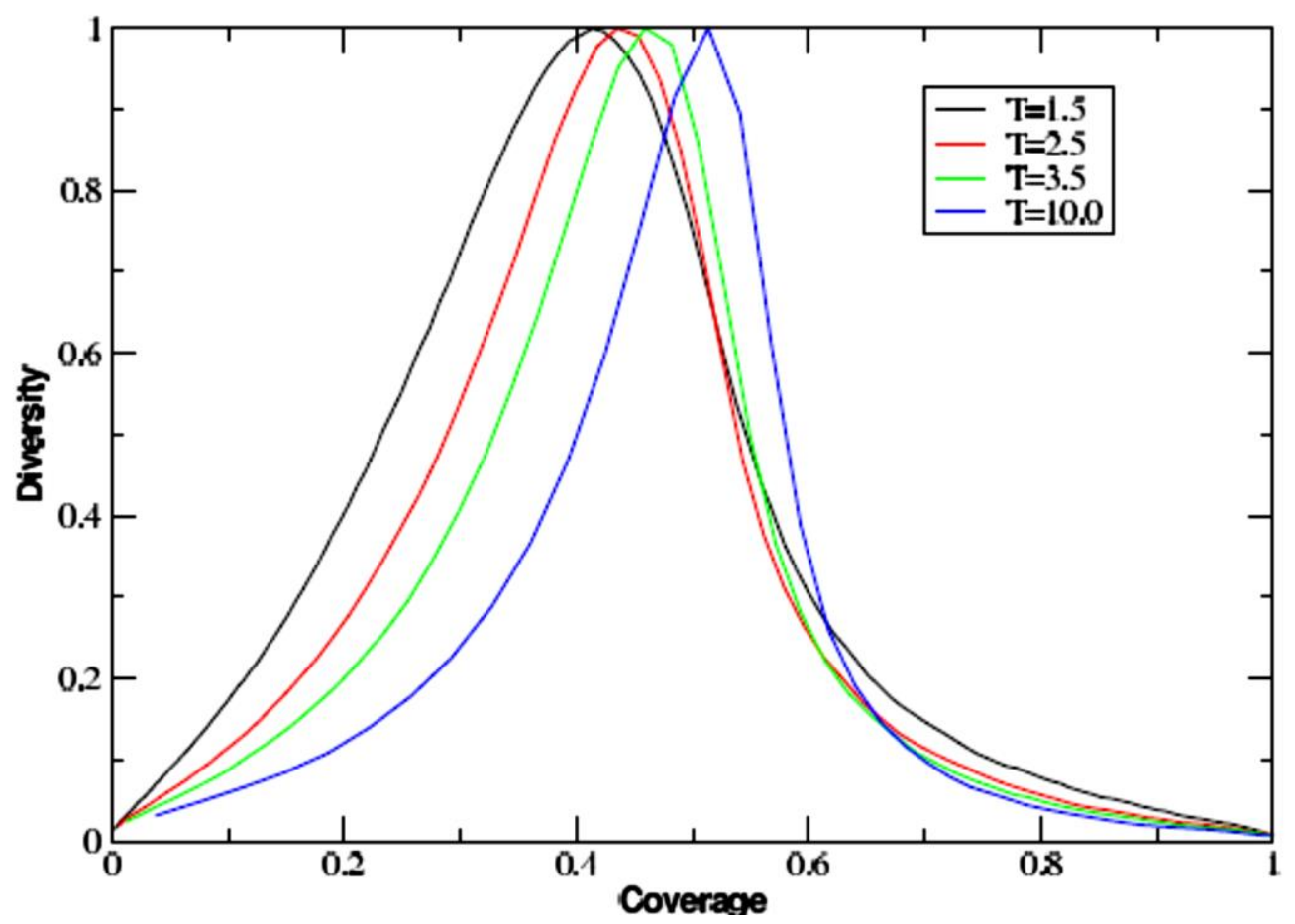

Figure 7: An example of $D(\theta)$ from a simulation with the simple rule, with $T=1.5,2.5,3.5,10.0$.

The diversity basically measure the total number of sizes. At lower temperatures, the maximum total number of sizes is reached faster, then it would quickly decrease again. As such, the growth model slows down in reaching the maximum of the rate of change. At higher temperature, the maximum number of size is slower to reach, which makes the system reach the maximum of the rate of change faster.

\section{Endnotes}

1 Alternatively we can record the coverage $\theta$ for every fixed interval of time and average the results. We obtain identical results either way. 


\section{References}

Frank, S., Roberts, D. E., and Rikvold, P. A. 2005. Effects of lateral diffusion on morphology and dynamics of microscopic lattice-gas model of pulsed electrodeposition. J. Chem. Phys 122, 064705 .

Hoshen, J. and Kopelman, R. 1976. Percolation and cluster distribution I: cluster multiple labeling technique and critical concentration algorithm. Phys. Rev. B 14, 3438.

Tsang, I. R. and Tsang, I. J. 1999. Cluster size diversity, percolation, and complex systems. Phys. Rev. E 60, 2684-2698.

Yang, C. N. and Lee, T. D. 1952. Theory of equations of state and phase transition II: lattice gas and ising model. Phys. Rev. 87, 404. 
This page is intentionally left blank. 